\title{
Surface Modification of Gold Nanoparticles with Polyhedral Oligomeric Silsesquioxane and Incorporation within Polymer Matrices
}

\author{
SHIAO-WEI KUO, ${ }^{1}$ YUNG-CHIEN WU, ${ }^{2}$ CHU-HUA LU, ${ }^{2}$ FENG-CHIH CHANG ${ }^{2}$ \\ ${ }^{1}$ Department of Materials and Optoelectronic Science, Center for Nanoscience and Nanotechnology, \\ National Sun Yat-Sen University, Kaohsiung, Taiwan \\ ${ }^{2}$ Department of Applied Chemistry, National Chiao Tung University, Hsinchu, Taiwan
}

Received 20 October 2008; revised 2 February 2009; accepted 2 February 2009

DOI: 10.1002 / polb.21686

Published online in Wiley InterScience (www.interscience.wiley.com).

\begin{abstract}
A new approach to achieve polymer-mediated gold ferromagnetic nanocomposites in a polyhedral oligomeric silsesquioxane (POSS)-containing random copolymer matrix has been developed. Stable and narrow distributed gold nanoparticles modified by 3-mercaptopropylisobutyl POSS to form Au-POSS nanoparticles are prepared by two-phase liquid-liquid method. These Au-POSS nanoparticles form partial particle aggregation by blending with poly( $n$-butyl methacrylate) (PnBMA) homopolymer because of poor miscibility between Au-POSS and PnBMA polymer matrix. The incorporation the POSS moiety into the PnBMA main chain as a random copolymer matrix displays well-dispersed gold nanoparticles because the POSS-POSS interaction enhances miscibility between gold nanoparticles and the PnBMA-POSS copolymer matrix. This gold-containing nanocomposite exhibits ferromagnetic phenomenon at room temperature. (C) 2009 Wiley Periodicals, Inc. J Polym Sci Part B: Polym Phys 47: 811-819, 2009
\end{abstract}

Keywords: nanocomposites; nanoparticles; POSS; self-assembly

\section{INTRODUCTION}

Metal and semiconductor nanoparticles possess unique electronic and chemical properties because of their high-surface areas and confinement of electronic states. ${ }^{1-4}$ The versatility of physical and chemical properties of these metal and semiconductor nanoparticles provides potential applications in fields ranging from optoelectronics, ${ }^{5}$ sensing, ${ }^{6}$ magnetism, ${ }^{7}$ catalysis, and medicine. ${ }^{8}$ Despite the diamagnetic character of bulk gold, well-dispersed gold nanoparticles capped with thi-

Correspondence to: S.-W. Kuo (E-mail: kuosw@faculty. nsysu.edu.tw)

Journal of Polymer Science: Part B: Polymer Physics, Vol. 47, 811-819 (2009)

(C) 2009 Wiley Periodicals, Inc. ols exhibit ferromagnetic behavior at room temperature. ${ }^{7}$ When gold nanoparticles are capped with thiols, the interaction between $\mathrm{Au}$ and the $\mathrm{S}$ atoms at the end of the alkyl chain modifies the electronic structure of the nanoparticle surface and results in $5 d$ localized holes. ${ }^{9}$ These holes produce the localized magnetic moments strongly aligned along the bond direction and thus create magnetic properties independent of temperature in the range $5-300 \mathrm{~K}^{10}$

Incorporation of nanoparticles into polymer matrices through nanoparticle-polymer matrix interaction is an important subject for materials engineering, therefore, various attempts have been attempted to achieve polymer-mediated nanocomposites. In general, three different 

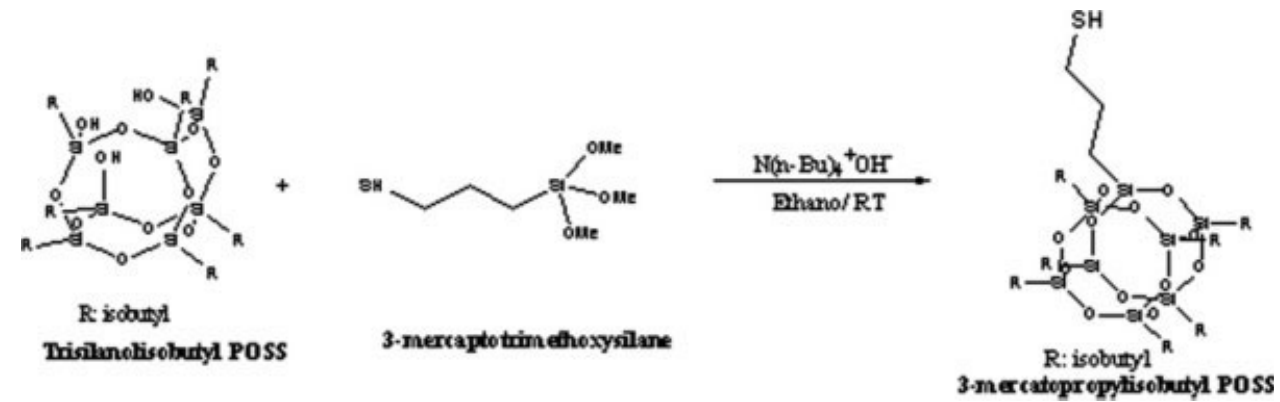

Scheme 1. Synthesis of 3-mercaptopropylisobutyl POSS macromer (SH-POSS).

approaches have been adapted to date. The first and less common technique involves polymerizing the matrix around the nanoparticles ${ }^{11}$ or stepwise assembly of a secondary polymer shell bound to the primary thiol through covalent or noncovalent (e.g., electrostatic or hydrogen bond) mode. ${ }^{12}$ The second technique consists of metallic cores included within the polymer matrix by functional groups of a capping polymer. ${ }^{13}$ These functionalized polymers can be obtained by polymerization of derivative monomers or postpolymerization funtionalization. ${ }^{14}$ The third approach involves the incorporation of surface-stabilized nanoparticles with polymer matrices. ${ }^{15,16}$ If the ligand of the gold nanoparticles is chemically identical or similar to the matrix, the particle incorporation is thermodynamically favorable. ${ }^{17}$

However, most of polymer/nanoparticles systems show strong particle aggregation because of poor miscibility between polymers and nanoparticles. In this study, these modified gold nanoparticles $(\mathrm{Au}-$ POSS) are compatible with $n$-butyl methyl methacrylate-co-polyhedral oligomeric silsesquioxane random copolymer (PnBMA-co-POSS) and result in well-distributed gold nanoparticles within the polymer matrix. ${ }^{18-21}$ To improve gold nanoparticles distribution within a polymer matrix, the general approach is to modify the nanoparticle surface to be compatible with polymer matrices. ${ }^{22-25}$

\section{EXPERIMENTAL}

\section{Syntheses of 3-Mercaptopropylisobutyl POSS and POSS-Modified Au Nanoparticles}

Trisilanolisobutyl polyhedral oligomeric silsesquioxane (T7-POSS) were obtained from Hybrid Plastics, Inc. 3-Mercaptopropylisobutyl POSS (SH-POSS) was prepared by the corner-capping method with hydroxyl groups on T7-POSS and methoxy groups on 3-methacryloxypropyl trime- thoxysilane in ethanol using tetrabutylammonium hydroxide as catalyst, as shown in Scheme 1. The POSS-modified gold nanoparticles (Au-POSS) used in this study was prepared through the concept of the standard Brust-Schiffrin methodology. ${ }^{22}$

\section{Syntheses of 3-(isobutyIPOSS)Propyl Methacrylate Momoner and Poly(n-butyl methacrylate-r-3- (isobutyIPOSS)propyl methacrylate) Random Copolymer (PnBMA-r-PiBPPMA)}

3-(isobutylPOSS)Propyl methacrylate (iBPPMA) was also prepared by corner-capping method with hydroxyl groups on T7-POSS $\AA$ and methoxyl groups on 3-methacryloxypropyl trimethoxysilane in ethanol with tetrabutylammonium hydroxide as catalyst. The synthesis of POSS-containing PnBMA random copolymers using conventional free radical polymerization has been described previously. ${ }^{26,27} 3$-iBPPMA and $n$-butyl methacrylate were dissolved in toluene and radical polymerization was carried out at $60{ }^{\circ} \mathrm{C}$ using AIBN as initiator, as shown in Scheme 2. According to the ${ }^{1} \mathrm{H}$ NMR spectrum and GPC traces, the polymer is denoted as $\mathrm{PnBMA}_{142}-r$-PiBPPMA 1 where 142 and 1 stand for the degree of polymerization $\left(M_{\mathrm{n}}=20,700\right.$, PDI $\left.=1.61\right)$. The same method was adopted to synthesize pure poly ( $n$-butyl methacrylate) $(\mathrm{PnBMA})\left(M_{\mathrm{n}}=27,000, \mathrm{PDI}=2.04\right)$.

\section{Preparation of Au-POSS/PnBMA-r-PiBPPMA Nanocomposites}

Polymer-mediated nanocomposites with gold nanoparticles were prepared by mixing POSSmodified gold nanoparticles (Au-POSS) with the $\mathrm{PnBMA}_{142}-r$-PiBPPMA ${ }_{1}$ copolymer in toluene. This mixture was stirred for $6-8 \mathrm{~h}$ and then the solvent was evaporated slowly at room temperature over 1 day. To ensure total elimination of solvent, the blend obtained was dried in a vacuum oven at $60^{\circ} \mathrm{C}$ for additional 2 days. 


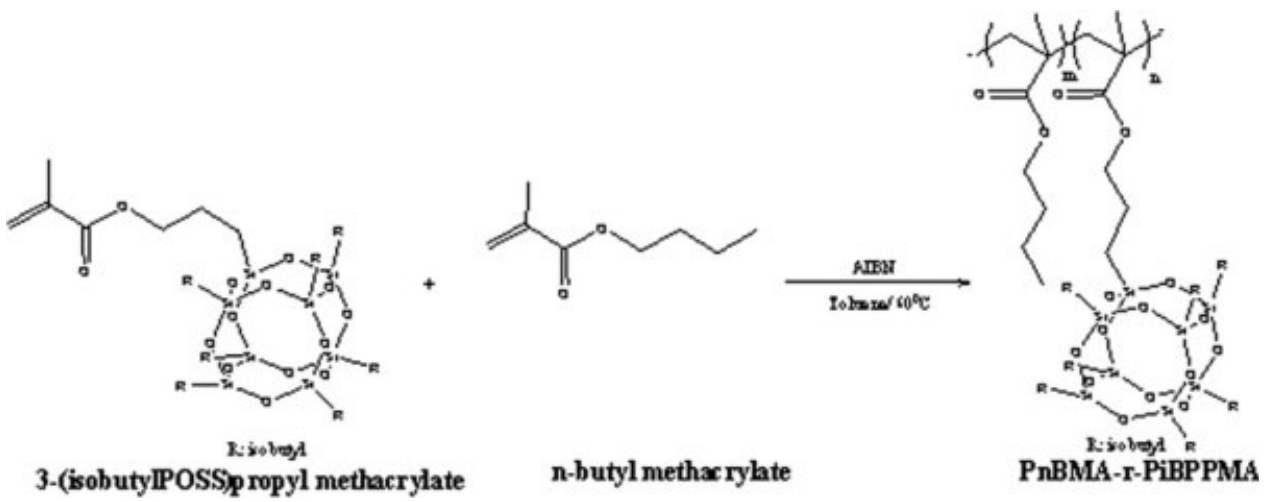

Scheme 2. Synthesis of PnBMA-r-PiBPPMA copolymer.

\section{Characterizations}

The intermolecular main chain spacing of the SHPOSS macromer and Au-POSS crystals were investigated using wide angle X-ray diffraction (XRD; M18XHF-SPA, MacScience Co., Japan). Diffractometry was carried out with a $\mathrm{Cu} \mathrm{K} \alpha$ radiation $(1.5418 \mathrm{E})$ with scan range of $2 \theta$ was $5^{\circ}-60^{\circ}$ with a step interval of $0.1^{\circ}$. The Au-POSS macro phase aggregation was observed by the optical microscopy (OM; DP12, OLYMPUS, Japan). The transmitted mode of OM was used to observe the image. Size distributions of the gold core in aggregation were measured by TEM (JEOL JEM1200CX II, JEOL JEM-200CX) image photograph. High resolution TEM (FEI Tecnai 20 G2 S-Twin, Philips) was used to observe size distributions. Sample for TEM were prepared by drop casting using one drop of solution onto copper grids (600 mesh) and dried in air overnight. UV-Vis spectra (DU700, Beckmann) were used to characterize gold nanoparticles aggregate phenomena from 450 to $750 \mathrm{~nm}$. The glass transition temperature was conducted on a TA Instrument DSC 2010 with scan rate of $20{ }^{\circ} \mathrm{C} / \mathrm{min}$ within the temperature range -100 to $100{ }^{\circ} \mathrm{C}$. Hysteresis loop measurements were performed using a commercial Superconducting Quantum Interference Device (SQUID) Magnetometer (MPMS5, USA) operating at 5 and $300 \mathrm{~K}$, with a maximum magnetic field of $10 \mathrm{kOe}$.

\section{RESULTS AND DISCUSSION}

\section{Crystalline Structure and Morphology of Au-POSS Nanoparticles}

Figure 1 displays the XRD pattern of (a) SHPOSS macromer and (b) Au-POSS nanoparticles.
Four peaks of (111), (200), (220), and (311) correspond to planes of a face-centered cubic (FCC) lattice of gold nanoparticles. The peak at the plane of (111) is more intense than others. The size of the $\mathrm{Au}$ nanoparticles is calculated by the following equation ${ }^{28}$ :

$$
t=\frac{0.9 \lambda}{B \cos \theta}
$$

where $t$ is particle size, $B$ is width in radians at intensity equal to half maximum intensity, the particle size obtained from Scherrer formula is about $4.8 \mathrm{~nm}$ in diameter. Major reflections (101), $(2 \overline{1} 0),(012)$ and $(\overline{1} 23)$ of the POSS crystal nearly disappear in Au-POSS nanoparticles except for those broad amorphous reflection signals because those large $\mathrm{Au}$ nanoparticles $(4.8 \mathrm{~nm}$ in diameter) disrupt the crystallization of the relatively smaller diameter of the SH-POSS crystalline

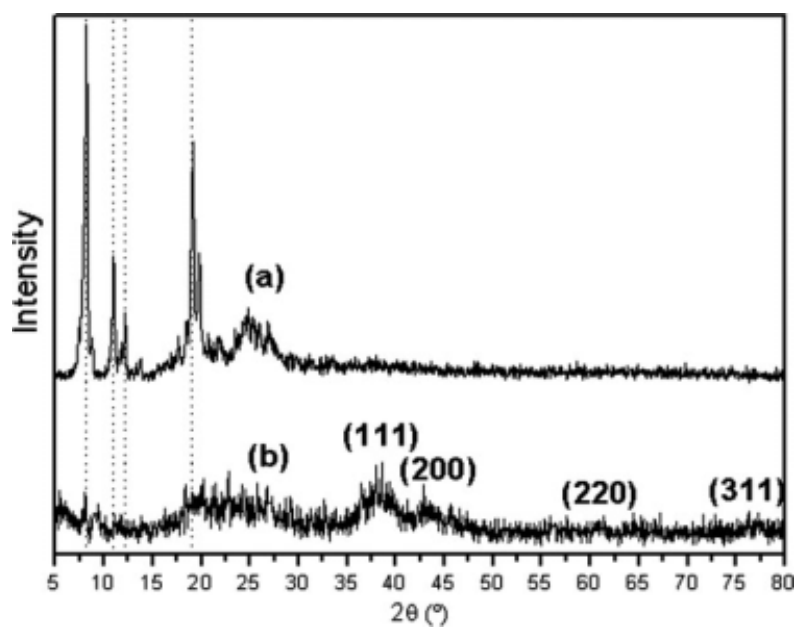

Figure 1. Powder diffractograms (XRD) of (a) SHPOSS macromer and (b) Au-POSS. 
$(\sim 1.5 \mathrm{~nm})$. It is clearly impossible for such large $\mathrm{Au}$ nanoparticles to be accommodated within the POSS crystalline structure, which is consistent with results reported by Waddon and coworkers. ${ }^{29}$ In their system, poleythylene- $b$-poly(norbornylene POSS) (PE- $b$-POSS) copolymers prepared by metallocene polymerization are able to afford the polyethylene backbone covalently attached to those bulky POSS nanoparticles, and the crystallization of PE is evidently limited by the presence of POSS groups on the side chain. They referred that the final microstructure may be controlled not only by the host chain topology but also by the competition between these two crystallization processes of PE and POSS.

Figure 2(a,b) shows the hydrodynamic diameter distributions of Au-POSS nanoparticles measured by dynamic light scattering analyses in toluene and tetrahydrofuran (THF), respectively. The hydrodynamic diameter of $\mathrm{Au}$ nanoparticles in toluene [Fig. 2(a)] presents a relatively narrower distribution about $9 \mathrm{~nm}$. Furthermore, Figure 2(b) indicates that these Au-POSS nanoparticles still possess relatively narrower distribution even after drying under vacuum at room temp and redissolving in another organic solvent (THF). This result demonstrates that the SH-POSS shell provides a stabilizing protective film of the Au-POSS nanoparticles during processes of drying and redissolving in different organic solvents

Figure 3(a) shows the OM image of Au-POSS aggregates, displaying the red ferny-like shape with the order of a few micrometers. The anisotropically shaped structure is similar to the crystalline SH-POSS except that the aggregation edges seem to be curved rather than flat. ${ }^{20}$ Figure 3(b) shows TEM image of $\mathrm{Au}$ nanoparticles dispersed within the ferny-like structure where these aggregates are comprised of individual gold nanoparticles surrounded by SH-POSS. The right-up inset of the Figure 3(b) shows the selected-area electron diffraction pattern of $\mathrm{Au}$ nanoparticles, revealing that these gold nanoparticles have FCC structure corresponding to (111), (200), and (220) gold crystalline facets while many small particles have independent orientations. High resolution of transmission electron microscopy (HRTEM) image of POSS-functionalized Au nanoparticles shown in Figure 3(c) displays two features: (1) the diameter of $\mathrm{Au}$ core calculated from Figure 3(c) is about $5 \mathrm{~nm}$, a close agreement with the diameter calculated by Scherrer formula as mentioned above by XRD pattern, and (2) the uniform core-core spacing is approximately equal
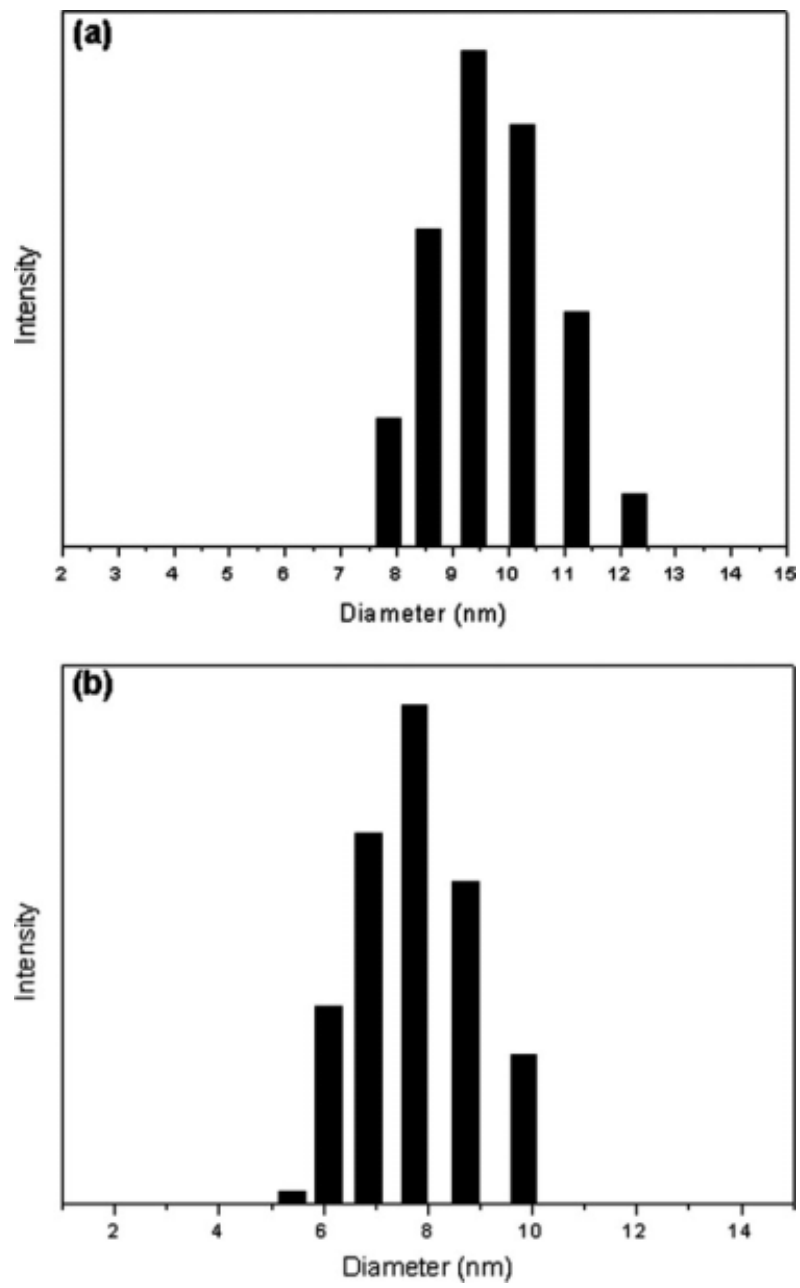

Figure 2. The hydrodynamic diameter distributions of Au-POSS nanoparticles in (a) toluene and replacing with (b) tetrahydrofuran (THF).

to about $8 \mathrm{~nm}$. Rotello and coworkers ${ }^{30}$ have reported that the well-defined spherical aggregates $(250 \mathrm{~nm} \sim 1.5 \mu \mathrm{m}$ diameter) with the uniform internal spacing are formed by using diaminopyridine-functionalized POSS nanoparticles and thymine functionalized $\mathrm{Au}$ nanoparticles. They reported that the center-to-center distance between the gold particles observed by SAXS is $3.65 \pm 0.08 \mathrm{~nm}$, corresponding to an edge-edge distance of about $2.1 \mathrm{~nm}$. This distance implies a side-to-side distance $(2.1 \mathrm{~nm})$ rather than face-toface distance $(\sim 4.5 \mathrm{~nm})$. In contrast to their results, this $3 \mathrm{~nm}$ surface to surface distance observed in our system indicates that face-to-face POSS-POSS packing interaction occurs rather than a side-to-side.

Journal of Polymer Science: Part B: Polymer Physics DOI $10.1002 /$ polb 


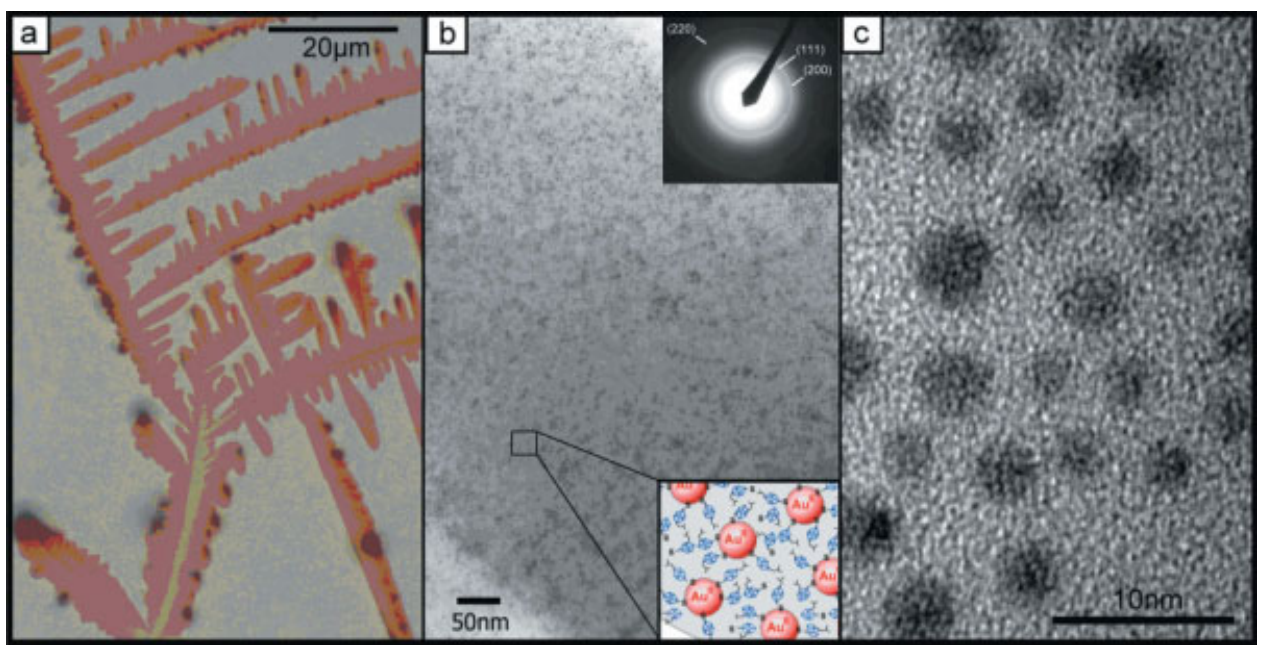

Figure 3. Micrographs of Au-POSS aggregation in the different scale by (a) optical microscopy (OM), (b) transmission electron microscopy (TEM), and (c) high resolution transmission electron microscopy (HRTEM). Insert in (b) show a electron diffraction pattern of Au-POSS nanoparticles and an aggregation model corresponding to a micrograph of (c).

\section{Incorporation of Au-POSS Nanoparticles into Polymer Matrices}

Figure 4 shows the morphology of Au-POSS nanoparticles incorporated into PnBMA homopolymer matrix. Most of these Au-POSS nanoparticles are located within the gray nano-sphere $(\sim 100 \mathrm{~nm}$ diameter) of SH-POSS domains and portions are dispersed in the PnBMA polymer matrix through the hydrophobic interaction between butyl groups of SH-POSS and butyl groups of PnBMA, as shown in the bottom inserted scheme. The gray nano-sphere, composed of the SH-POSS macromer, is formed by the strong POSS-POSS interactions between the SH-POSS from the desorption of thiol covalent bond (Au-S). Portion of Au nanoparticles surrounded by SH-POSS in the gray nano-sphere may connect each other to form twinned particles because of desorption of thiol covalent bond (shown in the inserted scheme). To improve the miscibility behavior in the Au-POSS/ PnBMA nanocomposite, the PnBMA matrix replaced by the PnBMA-r-PiBPPMA random copolymer which is expected to be more compatible with Au-POSS through both POSS-POSS and hydrophobic butyl interactions (shown in inset scheme of Fig. 5). Figure 5 demonstrates that these $\mathrm{Au}$ nanoparticles now are more evenly distributed within this random copolymer matrix than the PnBMA homopolymer matrix. However, if the number of grafts POSS in backbone of the random copolymer is increased, the increased unfavorable entropy quickly overcomes the enthalpy gain and the interfacial tension is actually increased, and thus induces more phase segregation. ${ }^{31,32}$

Wide-angle X-ray diffraction (WAXD) analyses were preformed to quantify the $d$-spacing of polymer chains when Au-POSS was incorporated. ${ }^{33}$ Figure 6 shows WAXD patterns of the PnBMA-rPiBPPMA random copolymer containing different contents of Au-POSS nanoparticles. The WAXD pattern of PnBMA-r-PiBPPMA random copolymer shows two amorphous halos, $2 \theta=6.5^{\circ}$ (a $d$-spacing of $13.4 \AA)$ and $2 \theta=17^{\circ}(d=5.3 \AA)$, respectively. These two halos are associated with distances between main chains $(d=13.4 \AA)$ and between isobutyl $(d=4.5 \AA)$, respectively. A sharp diffraction peak at $2 \theta=8^{\circ}(d=11.0 \AA)$ corresponds to small amount of free SH-POSS without bonding onto the surface of $\mathrm{Au}$ nanoparticles to form the crystalline SH-POSS macromer (101 hkl reflection of a rhombohedral lattice). The presence of Au-POSS nanoparticles shifts the amorphous halo at $2 \theta=6.5^{\circ}(d=13.4 \AA)$ to lower angle, implying that the introduction of Au-POSS nanoparticles expands intermolecular main-chain spacing.

The UV-Vis spectrum of a gold nanoparticle sample is a sensitive indicator of particle aggregation state. ${ }^{34}$ Figure 7 shows UV-Vis spectra of Au-POSS/PnBMA-r-PiBPPMA nanocomposites with various Au-POSS contents in toluene (300$800 \mathrm{~nm})$. The maxima absorption in toluene is at 


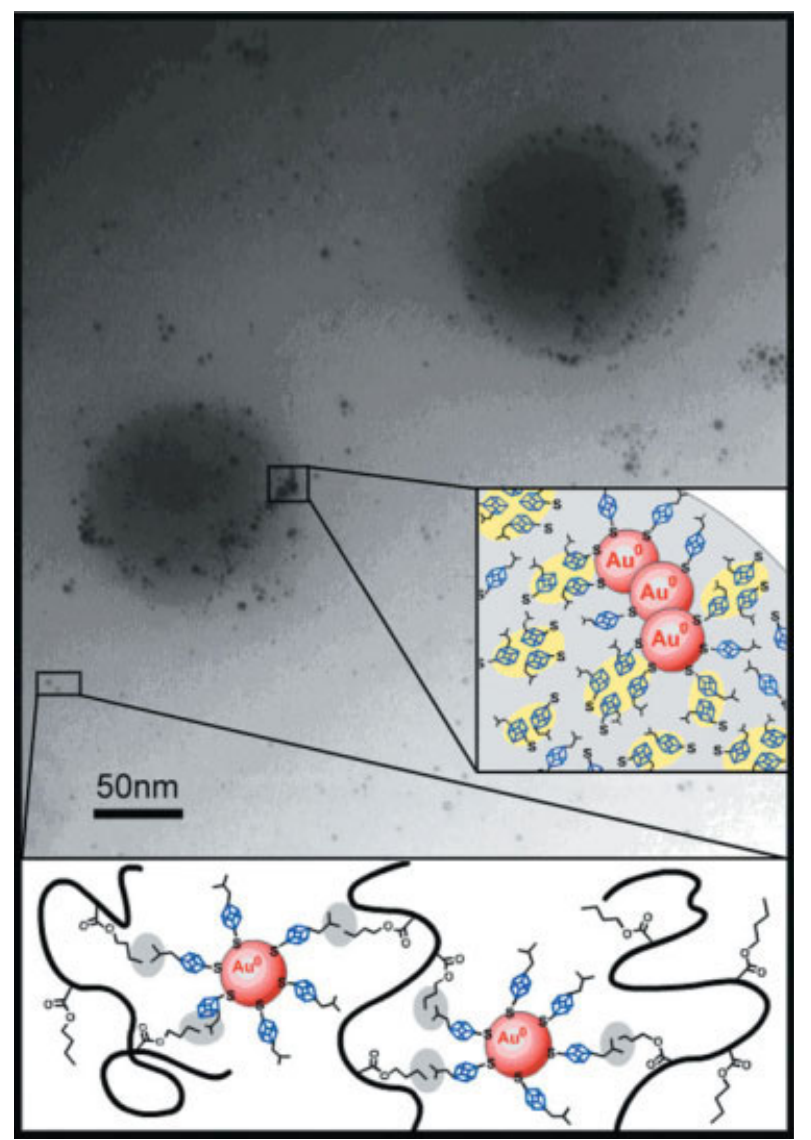

Figure 4. TEM images of Au-POSS nanoparticles in a PnBMA matrix, the inset scheme of (a) Au-POSS twining aggregation and (b) dispersed Au-POSS by hydrophobic interaction. [Color figure can be viewed in the online issue, which is available at www. interscience.wiley.com.]

$518 \mathrm{~nm}$ for $\mathrm{Au}$ nanoparticles, while the width of absorption is essentially same, independent of $\mathrm{Au}-\mathrm{POSS}$ nanoparticle content. In the framework of the Mie theory, ${ }^{35}$ the position of the maximum of the absorption band is determined by the refractive index of the medium surrounding the nanoparticles, whereas the width is controlled by the particle size. The observed invariant width reveals the absence of particle aggregation and particle edge-edge distances are the same. The same maxima absorption at $518 \mathrm{~nm}$ means the same dipole-dipole interaction, implying that desorption of Au-S thiolate covalent bond does not occur.

Figure 8 displays the differential scanningcalorimetric thermograms (DSC) of Au-POSS/ PnBMA-r-PiBPPMA nanocomposites with various

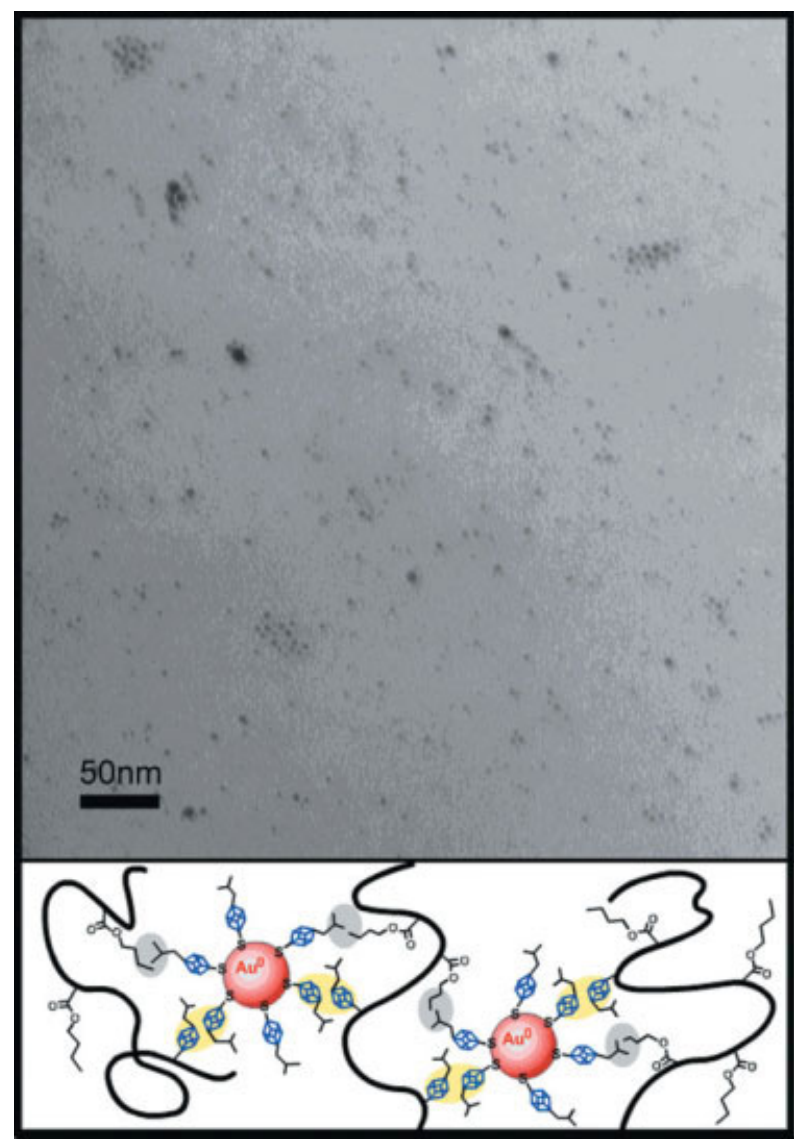

Figure 5. TEM images of Au-POSS nanoparticles in a PnBMA-r-PiBPPMA copolymer matrix, the inset scheme of well-dispersed Au-POSS nanoparticles by POSS-POSS interaction and hydrophobic interaction. [Color figure can be viewed in the online issue, which is available at www.interscience.wiley.com.]

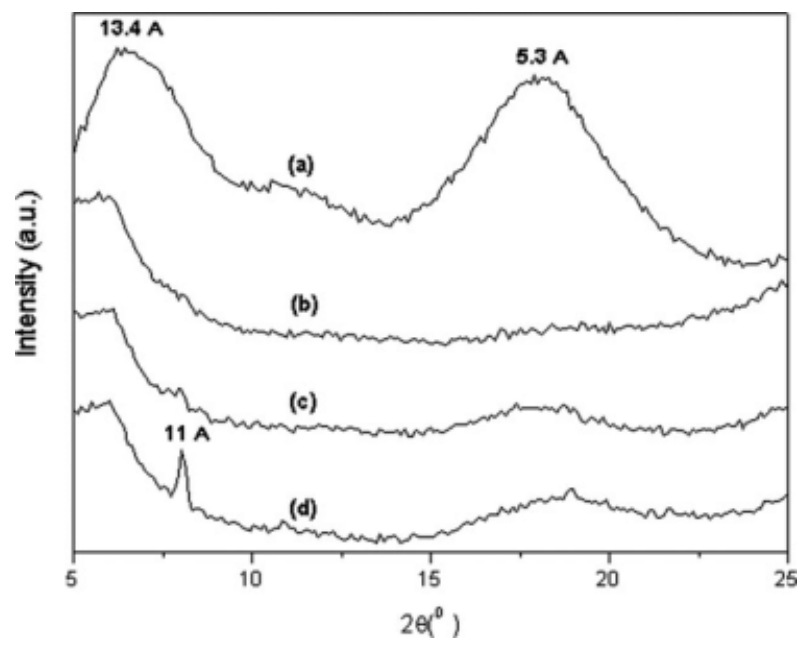

Figure 6. WAXD patterns of Au-POSS/PnBMA- $r$ PiBPPMA copolymer nanocomposites with different contents of Au-POSS nanoparticles: (a) 0, (b) 5, (c) 10, and (d) $20 \mathrm{wt} \%$.

Journal of Polymer Science: Part B: Polymer Physics DOI 10.1002/polb 


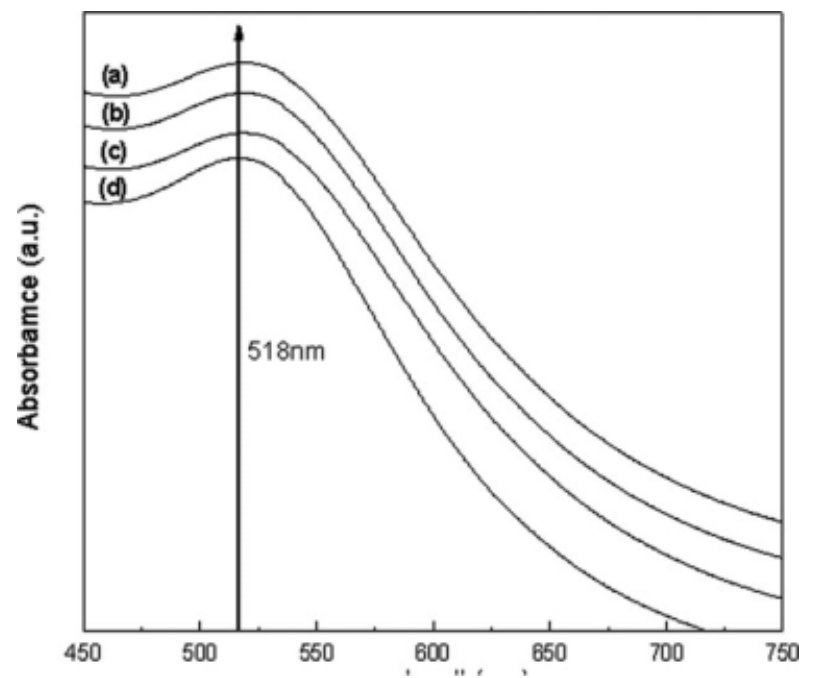

Figure 7. UV/Vis spectra of PnBMA-r-PiBPPMA copolymer nanocomposites with different contents of Au-POSS nanoparticles: (a) 5, (b) 10, (c) 20, and (d) 100 wt \% Au-POSS nanoparticles.

Au-POSS contents, revealing that the glass transition temperature monotonically decreases with increasing Au-POSS nanoparticles content. From the previous report, Haddad and coworkers ${ }^{33}$ reported that the change of the glass-transition temperature of POSS copolymers arises from three effects: (1) free volume, (2) steric barrier, and (3) POSS/POSS and polymer/polymer chain

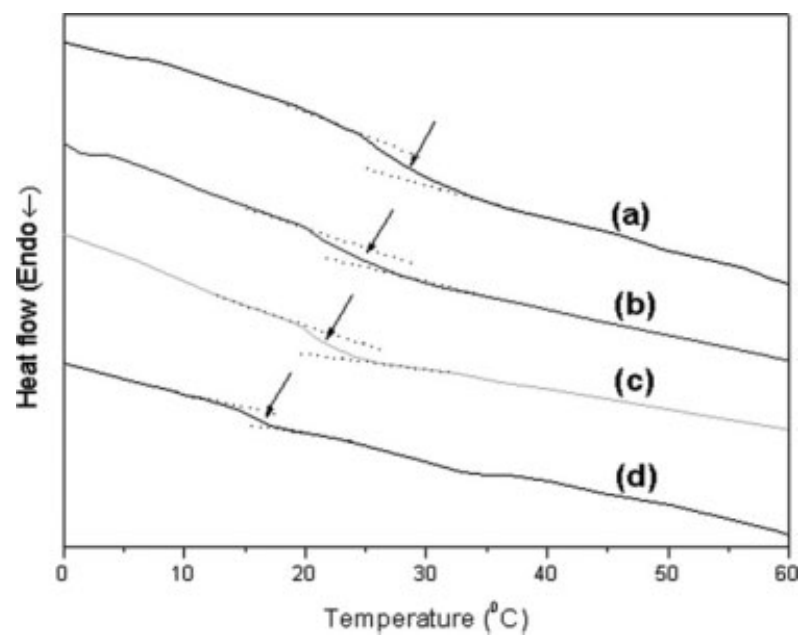

Figure 8. The second heating DSC thermograms of Au-POSS/PnBMA-r-PiBPPMA copolymer nanocomposites with different contents of Au-POSS nanoparticles: (a) 0, (b) 5, (c) 10, and (d) 20 wt \%.

Journal of Polymer Science: Part B: Polymer Physics DOI $10.1002 /$ polb

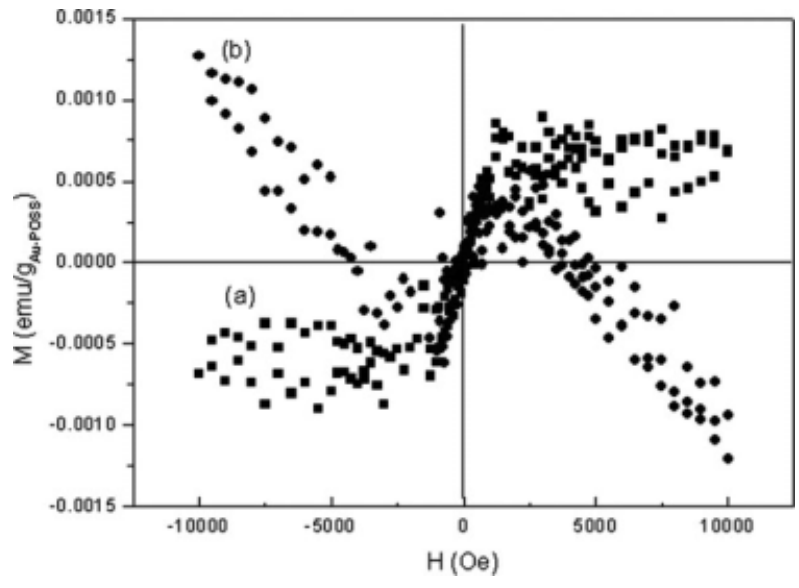

Figure 9. Hysteresis loops of Au-POSS/PnBMA-rPiBPPMA copolymer nanocomposites with $10 \mathrm{wt} \%$ of Au-POSS nanoparticles at (a) $5 \mathrm{~K}$ and (b) $300 \mathrm{~K}$. The magnetization is given in emu per gram of Au-POSS nanoparticles.

segmental interactions. ${ }^{36-38}$ XRD analyses shown in Figure 6 indicate that the bulky Au-POSS indeed increases the intermolecular distance between adjacent backbone chains, an indication of greater free volume and thus expected to be lower $T_{\mathrm{g}}$. The bulky Au-POSS tends to increase steric hindrance and induces higher $T_{\mathrm{g}}$. A strong segmental interaction is also in favor of higher $T_{\mathrm{g}}$, however, the POSS-POSS the hydrophobic butyl interactions are considered rather weak in present case. It appears that free volume increase of Au-POSS nanoparticles is dominant and acts as a plasticizer of the polymer matrix resulting in lower glass transition temperature.

Magnetic characterization of these samples was carried out with an SQUID magnetometer. ${ }^{39}$ Figure 9 shows the hysteresis loops of the 3-mercatopropylisobutyl POSS-capped Au nanoparticles $(10 \mathrm{wt} \%)$ in the copolymer matrix at 5 and $300 \mathrm{~K}$. The matrix of PnBMA-r-PiBPPMA presents a pure diamagnetic behavior without subtracting from the total magnetization. The most exciting feature from Figure 9 is that the thiol-capped $\mathrm{Au}$ nanoparticles can proceed magnetization process similarly to typical ferromagnetic materials, showing a hysteresis loop even up to room temperature. However, the magnetization of this nanocomposite is substantially lower than that previously reported for isolated thiol-capped $\mathrm{Au}$ nanoparticles with $1.4 \mathrm{~nm}$ diameter. ${ }^{7,40}$ Because the magnetic moment is come from the Au thiolate bond on the surface of the nanoparticle, 
larger $\mathrm{Au}$ nanoparticles has relatively smaller surface area and thus has lower magnetization. Electrons and holes of the $5 d$ band are itinerant reflecting the UV-Visible absorption spectrum as shown in Figure 7. Relative to the dodecanethiolcapped Au nanoparticles, POSS macromer bonded on Au nanoparticles creates higher projected area which tends to limit numbers of POSS macromers bonded on surface of $\mathrm{Au}$ nanoparticles. Fewer POSS macromers through thiol bonding on the $\mathrm{Au}$ nanoparticles result in expected lower ferromagnetic phenomena.

\section{CONCLUSIONS}

We have demonstrated that the uniform size distribution of the metal core of gold nanoparticles could be finely tuned by using POSS stabilizer molecules. The spacing between the Au nanoparticles was quantified a $\sim 3 \mathrm{~nm}$ POSS-POSS packing interaction. On the other hand, we have shown that random copolymers with functionalized POSS groups can be efficient at compatibilizing the immiscible behavior of $\mathrm{Au}$ nanoparticles and polymer matrix. Au nanoparticles would disperse in the matrix with a molecular level and absence of aggregates in the polymer matrix through this compatibilization function. We transfer the unique ferromagnetic property of Au nanoparticles into general polymer matrix to obtain magnetic polymer nanocomposites. The results shown in this article can be applied to other nanoparticles and polymer blend systems.

This work was supported by the National Science Council, Taiwan, Republic of China, under Contract No. NSC-97-2221-E-110-013-MY3 and NSC-97-2120M-009003 .

\section{REFERENCES AND NOTES}

1. El-Sayed, M. A. Acc Chem Res 2001, 34, 257-264.

2. Collier, C. P.; Vossmeyer, T.; Heath, J. R. Annu Phys Chem 1998, 49, 371-404.

3. Kim, H.; Daniels, E. S.; Dimonie, V. L.; Klein, A. J Polym Sci Part A: Polym Chem 2008, 46, 912925.

4. Han, D. H.; Pan, C. Y. J Polym Sci Part A: Polym Chem 2008, 46, 341-352.

5. Trindade, T.; Brien, P. O.; Pickett, N. L. Chem Mater 2001, 13, 3843-3849.
6. Krasteva, N.; Besnard, I.; Guse, B.; Bauer, R. E.; Mullen, K.; Yasuda, A.; Vossmeyer, T. Nano Lett 2002, 2, 55-57.

7. Crespo, P.; Litran, R.; Rojas, T. C.; Multigner, M.; de a Fuente, J. M.; Sanchez-Lopez, J. C.; Garcia, M. A.; Hernando, A.; Penades, S.; Fernandez, A. Phys Rev Lett 1999, 82, 3264-3267.

8. Daniel, M. C.; Astruc, D. Chem Rev 2004, 104, 293-346.

9. Zhang, P.; Sham, T. K. Phys Rev Lett 2003, 90, 245502

10. Carmeli, I.; Leitus, G.; Naaman, R.; Reich, S.; Vager, Z. J. Chem Phys 2003, 118, 372-378.

11. Mandal, T. K.; Fleming, M. S.; Walt, D. R. Nano Lett 2002, 2, 3-7.

12. Gittins, D. I.; Caruso, F. J. Phys Chem B 2001, 105, 6846-6852.

13. Boal, A. K.; Ilhan, F.; DeRouchey, J. E.; ThurnAlbrecht, T.; Russell, T. P.; Rotello, V. M. Nature 2000, 404, 746-748.

14. Shenhar, R.; Norsten, T. B.; Rotello, V. M. Adv Mater 2005, 17, 657-669.

15. Corbierre, M. K.; Cameron, N. S.; Sutton, M.; Mochrie, S. G. J.; Lurio, L. B.; Ruhm, A.; Lennox, R. B. J Am Chem Soc 2001, 123, 1041110412.

16. Bockstaller, M. R.; Lapetnikov, Y.; Margel, S.; Thomas, E. L. J Am Chem Soc 2003, 125, 52765277.

17. Cowie, J. M. G. Polymers: Chemistry and Physics of Modern Materials, 2nd ed.; Blackie Academic \& Professional: New York, 1991.

18. Brust, M.; Walker, M.; Bethell, D.; Schiffrin, D. J.; Whyman, R. J Chem Soc: Chem Commun 1994, 801-802.

19. Dishner, M. H.; Hemminger, J. C.; Feher, F. J. Langmuir 1996, 12, 6176-6178.

20. Schmid, G.; Pugin, R.; Malm, J. O.; Bovin, J. O. Eur J Inorg Chem 1998, 1998, 813-817.

21. Carroll, J. B.; Frankamp, B. L.; Srivastava, S.; Rotello, V. M. J Mater Chem 2004, 14, 690694.

22. Yonezawa, T.; Yasui, K.; Kimizuka, N. Langmuir 2001, 17, 271-273.

23. Waddon, A. J.; Coughlin, E. B. Chem Mater 2003, 15, 4555-4561.

24. Zhang, W.; Fu, B. X.; Seo, Y.; Schrag, E.; Hsiao, B.; Mather, P. T.; Yang, N. L.; Xu, D.; Ade, H.; Rafailovich, M.; Sokolov, J. Macromolecules 2002, 35, 8029-8038.

25. Jewrajka, S. K.; Chatterjee, U. J Polym Sci Part A: Polym Chem 2006, 44, 1841-1854.

26. Lichtenhan, J. D.; Otonari, Y. A.; Carr, M. J. Macromolecules 1995, 28, 8435-8437.

27. Pyun, J.; Matyjaszewski, K. Macromolecules 2000, 33, 217-220.

28. Cullity, B. D. Elements of X-ray Diffraction; Addision-Wesley: Reading, MA, 1978; p 102.

Journal of Polymer Science: Part B: Polymer Physics DOI $10.1002 /$ polb 
29. Zheng, L.; Waddon, A. J.; Farris, R. J.; Coughlin, E. B. Macromolecules 2002, 35, 2375-2379.

30. Carroll, J. B.; Frankamp, B. L.; Rotello, V. M. Chem Commun 2002, 1892-1893.

31. Lyatskaya, Y.; Gersappe, D.; Gross, N. A.; Balazs, A. C. J Phys Chem 1996, 100, 1449-1458.

32. Ge, S.; Guo, L.; Rafailovich, M.; Sokolov, J.; Overney, R.; Buenviajie, C.; Peiffer, D.; Schwarz, S. Langmuir 2001, 17, 1687-1692.

33. Wu, J.; Haddad, T. S.; Kim, G. M.; Mather, P. T. Macromolecules 2007, 40, 544-554.

34. Kim, Y.; Johnson, R. C.; Hupp, J. T. Nano Lett 2001, 1, 165-167.
35. Hovel, H.; Fritz, S.; Hilger, A.; Kreibig, U.; Vollmer, M. Phys Rev B 1993, 48, 178-185.

36. Xu, H.; Kuo, S. W.; Lee, J. S.; Chang, F. C. Macromolecules 2002, 35, 8788-8793.

37. Xu, H.; Kuo, S. W.; Lee, J. S.; Chang, F. C. Polymer 2002, 43, 5117-5124.

38. Chan, S. C.; Kuo, S. W.; She, H. S.; Lee, H. F.; Chang, F. C. J Polym Sci Part A: Polym Chem 2007, 45, 125-135.

39. Guschl, P. C.; U.S. Patent 2004, 7,390,579.

40. de la Venta, J.; Pucci, A.; Pinel, E. F.; Garcia, M. A.; Fernandez, C. J.; Crespo, P.; Mazzoldi, P.; Ruggeri, G.; Hernando, A. Adv Mater 2007, 19, 875-877. 